\title{
Combined Association Between ADIPOQ, PPARG, and TNF Genes Variants and Obstructive Sleep Apnea in Chinese Han Population
}

\author{
Juan Li',2, Qianwen Lv', Haili Sun (D)', Yunyun Yang',3, Xiaolu Jiao',3, Song Yang' ${ }^{1, \text { Huahui Yu }}{ }^{1,3}$, \\ Yanwen Qin (1D) ${ }^{1,3}$
}

'Key Laboratory of Upper Airway Dysfunction-Related Cardiovascular Diseases, Beijing An Zhen Hospital, Capital Medical University, Beijing Institute of Heart, Lung, and Blood Vessel Diseases, Beijing, 100029, People's Republic of China; ${ }^{2}$ Emergency Department, Beijing Shijitan Hospital, Capital Medical University, Beijing, 100038, People's Republic of China; ${ }^{3}$ Key Laboratory of Remodeling-related Cardiovascular Diseases, Beijing An Zhen Hospital, Capital Medical University, Beijing Institute of Heart, Lung and Blood Vessel Diseases, Beijing, I00029, People's Republic of China

Correspondence: Yanwen Qin, Key Laboratory of Upper Airway Dysfunction-Related Cardiovascular Diseases, Beijing An Zhen Hospital, Capital Medical University, Beijing Institute of Heart, Lung and Blood Vessel Diseases, No. 2 Anzhen Road, Chaoyang District, Beijing, I00029, People's Republic of China, Tel +86-10-64456529, Fax+86-10-64456095, Email qinyanwen@vip.126.com; qinyanwen@ccmu.edu.cn

Purpose: Obstructive sleep apnea (OSA) is a common chronic polygenic disease. Multiple genetic markers associated with OSA have been identified by genome-wide association studies. Here, we aimed to construct a polygenic risk score (PRS) and examine the association with the presence of OSA in a Chinese Han Population.

Patients and Methods: This study included 1057 individuals who were genotyped for nine susceptibility loci from three genes ( $A D I P O Q, P P A R G$, and TNF), from which each individual's PRS was calculated by summing the number of risk alleles. The associations between PRS and OSA were determined by logistic regression analyses. Model discrimination was assessed by a receiver operating characteristic (ROC) curve using bootstrapping with 1000 resamples.

Results: The subjects included 874 with OSA and 183 controls. A higher PRS was associated with an increased apnea-hypopnea index (AHI). The PRS was an important risk factor for the development of OSA (OR $=1.237$ per SD, $P=0.030)$. Subjects with higher PRS had a 2.88 -fold (95\% CI: $1.393-5.955, P=0.004)$ and 5.402-fold (95\% CI: $2.311-12.624, P<0.001)$ greater risk for having OSA and moderate-to-severe OSA, respectively, compared with those with lower genetic risk. More importantly, compared with determination of risk based solely on clinical factors, addition of the PRS increased discriminatory accuracy for both OSA (AUC from 0.75 to $0.78, P=0.02$ ) and moderate-to-severe OSA (AUC from 0.80 to $0.83, P=0.02$ ).

Conclusion: Our study suggests that the PRS is independently associated with AHI and OSA. Combining PRS with conventional risk factors could improve the discrimination of OSA.

Keywords: polygenic risk score, apnea-hypopnea index, single nucleotide polymorphisms, discriminatory accuracy

\section{Introduction}

Obstructive sleep apnea (OSA) is a common sleep disorder caused by blockage of the Upper Airway. ${ }^{1}$ Patients with OSA experience recurrent episodes of intermittent hypoxia and hypercapnia during sleep. ${ }^{2}$ Although $34 \%$ of men and $17 \%$ of women are estimated to be affected by this disease, the majority of OSA patients remain largely undiagnosed. ${ }^{3}$ Moreover, OSA itself has been recognized as a risk factor for cardiovascular disease, metabolic dysregulation, cancer, and all-cause death. ${ }^{4}$ OSA is a complex disorder involving multiple factors, including hereditary and environmental components. ${ }^{5}$ There is growing evidence that genetic factors and their interactions with the environment can influence the development of OSA. Indeed, most cases of OSA do not exhibit classical Mendelian patterns of inheritance, suggesting that multiple genes are involved in its pathogenesis. ${ }^{6}$

Obesity, especially abdominal obesity, is a major risk factor for OSA. ${ }^{7}$ In obese patients, adipocytes secrete a variety of adipokines and inflammatory factors, such as adiponectin (ADIPOQ), peroxisome proliferator-activated receptor 
gamma (PPARG), and tumor necrosis factor (TNF). These factors play an important role in the occurrence and progression of OSA, as well as in the development of related complications. ${ }^{8,9}$ There is evidence of both direct genetic contributions to OSA susceptibility and indirect contributions such as obesity, craniofacial structure, neurological control of upper airway muscles and of sleep and circadian rhythm. Thus, common polymorphisms in genes involved in obesity are possible good candidates for predicting OSA susceptibility.

Most studies on OSA genetics have focused on single genes. A genome-wide approach may provide greater information, beyond that of individual candidate genes. However, data from such studies are limited at present. We previously showed that $A D I P O Q$ variants rs3774262, rs4686803, rs1063537, rs2082940, and the PPARG variant rs1801282 were associated with the prevalence of OSA in Han Chinese individuals. ${ }^{10,11}$ Using the same dataset, we also found that single nucleotide polymorphisms (SNPs) rs3093661, rs3093662, and rs3093664 in the TNF gene were associated with increased risk of OSA (Supplemental Table 1).

Polygenic risk scores (PRS), which consider multiple SNPs associated with diseases, have been widely studied in many conditions, such as coronary heart disease, cancer, and type 2 diabetes. However, it has not yet been applied to OSA disease. Therefore, we combined nine SNPs from ADIPOQ, PPARG, and TNF genes into a PRS, then determined the effects of the PRS on apnea-hypopnea index (AHI) and the risk of OSA in 1057 individuals.

\section{Methods}

\section{Study Participants}

Subjects included in this study were Chinese individuals who visited the Beijing An Zhen Hospital because of snoring or sleep apnea from April 2017 to January 2019. All participants received an overnight polysomnography (PSG), which was conducted using a level II portable diagnostic device (SOMNOscreen; SOMNOmedics GmbH, Randersacker, Germany) approved by the US Food and Drug Administration. This included nasal pressure sensors, thermistors, thoracoabdominal belts, blood oxygen probes, and snoring sensors to monitor breathing, as described in our first study. ${ }^{12}$ Ventilatory flow at the nose and mouth was measured with thermistors. Ventilatory movements of the chest and abdomen were monitored by inductive plethysmography bands. The arterial oxygen saturation $(\mathrm{SpO} 2)$ was measured transcutaneously with fingertip pulse oximetry. We applied the scoring guidelines and sleep apnea definition of the American Academy of Sleep Medicine. A respiratory event was scored as an apnea in adults if complete cessation of airflow occurred for $\geq 10 \mathrm{~s}$. Hypopnea was defined as respiratory airflow reduced by $30 \%$ with a $4 \%$ decrease in oxygen saturation. Apnea events were classified as obstructive, mixed, or central according to the presence or absence of breathing efforts with thoracoabdominal paradox. The AHI was determined by dividing the total number of apnea/hypopnea events by the estimated hours of sleep. OSA patients and non-OSA subjects were confirmed on the basis of American Academy of Sleep Medicine Guidelines. ${ }^{13}$ OSA was defined as those subjects with an AHI $\geq 5$ per hour. Subjects with an AHI $<5$ served as controls. OSA severity was quantified via the AHI: mild OSA (AHI: 5-14.9), moderate OSA (AHI: 15-29.9), or severe OSA (AHI $\geq 30$ ). The criteria for exclusion from the study included: (1) the presence of other sleep disorders or a history of treatment for sleep-related breathing disorders; (2) the presence of other diseases, such as congestive heart failure, cancer, acute infectious disease, hepatic dysfunction, or abnormal renal function; and (3) pregnancy. In total, 1057 subjects were included in the study (the study flow chart is shown in Supplementary Figure 1), and all participants provided written informed consent. The study was approved by the Medicine Ethics Committee of Beijing An Zhen Hospital (2017005) and was registered in the Chinese Clinical Trial Register (ChiCTR-ROC-17011027).

\section{Data Collection}

Demographic data, including age, gender, height, body weight, smoking status, drinking status, and current medication usage, were collected using questionnaires and medical histories. Participants also completed the Berlin questionnaire (BQ) and the Epworth Sleepiness Scale questionnaire. Patients were considered high risk for OSA if they scored positive in two or more categories of the $\mathrm{BQs},{ }^{14}$ while the remaining individuals were considered low risk. Subjects were classified as nonsmokers if they had never smoked or had stopped smoking $\geq 1$ year before enrollment in the study; all other subjects were classified as smokers. Subjects were defined as drinkers if they drink $\geq 3$ times a week. Obesity was 
defined as a body mass index (BMI) $\geq 28 \mathrm{~kg} / \mathrm{m}^{2}$, according to the recommendations of the Health Promotion Administration. ${ }^{15}$ Blood samples were taken after $8-12 \mathrm{~h}$ of fasting from each subject and centrifuged at $2943 \times \mathrm{g}$ at $4^{\circ} \mathrm{C}$ for $10 \mathrm{~min}$. Half the sample was used for DNA isolation, while the remaining half was used to obtain serum for biochemical analysis. Genomic DNA was extracted from $200 \mu \mathrm{L}$ of blood according to the protocol provided in the QIAamp DNA Mini Kit (Qiagen, Hilden, Germany). Fasting plasma glucose (FPG) levels and a fasting lipid profile, including the levels of high-density lipoprotein cholesterol (HDL-C), low-density lipoprotein cholesterol (LDL-C), total cholesterol (TC), and triglycerides (TGs), were measured according to standard laboratory methods at Beijing An Zhen Hospital.

\section{Genotyping and Calculation of PRS}

We selected nine SNPs for the PRS from obesity-related genes, which had reported strong associations with OSA based on the literature and our previous work. These nine SNPs in three genes (ADIPOQ rs1063537, ADIPOQ rs2082940, $A D I P O Q$ rs4686803, ADIPOQ rs2241766, ADIPOQ rs3774262, TNF rs3093661, TNF rs3093662, TNF rs3093664, and PPARG rs 1801282) chosen for this study were genotyped using Agena MassARRAY (Agena Bioscience, San Diego, CA, USA) following the manufacturer's protocol. Assay Designer version 4.0 was used in accordance with the manufacturer's guide to determine the specific PCR primer and extension primer sequences (Supplemental Table 2). Agena Typer 4.0 software was used for data management and analysis. SNPs with $P$-values for the test of deviation from the HardyWeinberg equilibrium of 0.05 were excluded for further analysis.

Individuals homozygous for non-risk alleles were given a score of 0 , heterozygotes were given a score of 1 , and individuals homozygous for risk alleles were given a score of 2 . The PRS was constructed by summing the number of risk alleles among the nine gene variants in each individual.

\section{Statistical Analyses}

Continuous variables are expressed as the mean \pm standard deviation or median (interquartile range), and categorical variables are expressed as a number (percentage). Differences in the distribution of risk factor data between OSA and controls were assessed using a two-sided $\chi^{2}$ test or a Student's $t$-test, where appropriate. Each allele-specific odds ratio (OR) with 95\% confidence intervals (CIs) and $P$-values for OSA were calculated under the assumption of an additive model using logistic regression analysis, adjusting for age, sex, BMI, systolic blood pressure (SBP), diastolic blood pressure (DBP), TG, FPG, and smoking status. Association between the PRS and AHI was tested with linear regression. The risk of OSA associated with the 9-SNP PRS was calculated continuously. This was done with the addition of one risk allele. Then, after stratification into low risk ( $\leq 20$ th percentile), intermediate risk (20th-80th percentile), and high risk score ( $\geq 80$ th percentile) categories by calculating ORs, it was adjusted for clinical risk factors. Univariate and multivariate logistic regression analysis was performed to identify variables $(P<0.05)$ that significantly affected OSA. We then estimated the area under the receiver operating characteristic (ROC) curve (AUC) for risk scores that included clinical risk factors alone, the PRS alone, and the two combined. To compare the AUC of two models, we calculated the Wald statistic by dividing the observed difference of AUC estimates of the two models by the standard error of the difference obtained from bootstrapping. The model was validated internally using bootstrapping with 1000 resamples. We considered a two-sided $P$-value $<0.05$ as statistically significant. Analyses were carried out using SPSS 23.0 (IBM Corp., Armonk, NY, USA) and EmpowerStats software (www.empowerstats.com, X\&Y solutions, Inc., Boston, MA, USA).

\section{Results}

\section{Physical and Clinical Characteristics of Study Subjects}

We evaluated a total of 1057 Chinese subjects, including 874 with OSA and 183 controls. Their physical and clinical characteristics are presented in Table 1. In brief, individuals with OSA had a higher average BMI $(P<0.001)$ than the controls, and those with OSA were older than the controls $(P=0.026)$. There were more males $(P<0.001)$ in the OSA group compared with the controls. OSA patients tended to have higher levels of TG $(P<0.001)$. HDL-C levels $(P=$ 
Table I Characteristics of Participants

\begin{tabular}{|c|c|c|c|}
\hline \multirow[t]{2}{*}{$\mathbf{N}$} & Control & OSA & \multirow[t]{2}{*}{$\mathbf{P}$} \\
\hline & 183 & 874 & \\
\hline Age (years) & $50.4 \pm 14.3$ & $52.7 \pm 12.2$ & $0.026 *$ \\
\hline Male (n, \%) & 123 (67.2\%) & 738 (84.4\%) & $<0.001 * *$ \\
\hline BMI $\left(\mathrm{kg} / \mathrm{m}^{2}\right)$ & $24.6 \pm 3.9$ & $27.8 \pm 3.9$ & $<0.001^{* *}$ \\
\hline SBP (mmHg) & $126.9 \pm 19.7$ & $|3| .2 \pm 18.7$ & $0.005^{*}$ \\
\hline $\mathrm{DBP}(\mathrm{mmHg})$ & $78.0 \pm 14.3$ & $81.5 \pm 14.0$ & $0.003^{*}$ \\
\hline $\mathrm{HDL}-\mathrm{C}(\mathrm{mmol} / \mathrm{L})$ & $1.2 \pm 0.3$ & $1.2 \pm 0.8$ & 0.584 \\
\hline LDL-C(mmol/L) & $2.7 \pm 0.8$ & $2.7 \pm 1.0$ & 0.928 \\
\hline TC (mmol/L) & $4.5 \pm 1.1$ & $4.6 \pm 2.3$ & 0.532 \\
\hline $\mathrm{TG} \#(\mathrm{mmol} / \mathrm{L})$ & $1.29(0.95-1.72)$ & $1.62(1.13-2.31)$ & $<0.001^{* *}$ \\
\hline FPG $(\mathrm{mmol} / \mathrm{L})$ & $5.4 \pm 1.7$ & $6.6 \pm 20.4$ & 0.405 \\
\hline Drinker (n, \%) & 71 (38.8\%) & $372(42.6 \%)$ & 0.342 \\
\hline Smoker (n, \%) & 70 (38.3\%) & 397 (45.7\%) & 0.066 \\
\hline $\mathrm{AHI}$ (times/h) & $2.3(1.3-3.4)$ & $22.9(12.4-40.1)$ & $<0.00 I^{* *}$ \\
\hline ESS score\# & $6(3-10)$ & $7(4-12)$ & $<0.001^{* *}$ \\
\hline Berlin Questionnaire high risk (n, \%) & 35 (27.78\%) & $458(56.68 \%)$ & $<0.00 I^{* *}$ \\
\hline
\end{tabular}

Notes: Values were expressed as mean \pm standard deviation, median \pm interquartile range or $\mathrm{n}(\%)$. \#Data were asymmetrically distributed and expressed as median \pm interquartile. Differences between groups were analyzed by independent Student's $t$-test, Fisher's exact test, $\chi^{2}$ test, or Wilcoxon test. $* \mathrm{P}<0.05$, $* * \mathrm{P}<0.001$.

Abbreviations: OSA, obstructive sleep apnea; BMI, body mass index; SBP, systolic blood pressure; DBP, diastolic blood pressure; FPG, fasting plasma glucose; TG, triglycerides; TC, total cholesterol; LDL-C, low-density lipoprotein cholesterol; HDL-C, high-density lipoprotein cholesterol; AHI, apnea-hypopnea index; ESS, Epworth Sleepiness Scale.

0.584), LDL-C levels $(P=0.928)$, and TC levels $(P=0.532)$ showed no significant differences between the two groups. Blood pressure measurements in control subjects were significantly different from those of OSA patients (all $P<0.05$ ). The OSA patient group tended to have more smokers than the control group, but the difference was not statistically significant $(P=0.066)$.

\section{Association of PRS with $\mathrm{AHI}$ and OSA}

The genotype distributions of these nine SNPs were all in Hardy-Weinberg equilibrium (Supplemental Table 3). The associations of each of the nine SNPs with OSA are shown in Table 2. When analyzed individually, each SNP showed little or no significant association with OSA.

The PRS was independently associated with linear increases in AHI (Table 3). An increase of 1 SD of the PRS was associated with an increase of 1.24 times/h $(P=0.046)$ in AHI. Higher PRS was associated with higher AHI. The medians and 75th percentiles of AHI increased significantly from lower PRS to higher PRS (Figure 1).

Table 2 Individual Effects of 9 Risk Alleles of the Susceptibility Loci on OSA

\begin{tabular}{|c|c|c|c|c|c|}
\hline Gene & SNP & Risk Allele & OR (95\% Cl) & $\mathbf{P}$ & FDR \\
\hline ADIPOQ & rs 1063537 & $\mathrm{~T}$ & $1.305(0.959-1.776)$ & 0.091 & 0.0278 \\
\hline ADIPOQ & rs2082940 & $\mathrm{T}$ & $1.284(0.945-1.744)$ & 0.110 & 0.0333 \\
\hline ADIPOQ & rs4686803 & $\mathrm{T}$ & $1.335(0.981-1.817)$ & 0.066 & 0.0167 \\
\hline ADIPOQ & rs224I766 & G & I.342(0.985-I.827) & 0.062 & 0.0111 \\
\hline ADIPOQ & rs3774262 & A & $1.329(0.976-1.811)$ & 0.071 & 0.0222 \\
\hline$T N F$ & rs309366I & A & $1.394(0.685-2.837)$ & 0.359 & 0.05 \\
\hline$T N F$ & rs3093662 & G & $1.493(0.735-3.030)$ & 0.268 & 0.0389 \\
\hline TNF & rs3093664 & G & $1.442(0.709-2.932)$ & 0.312 & 0.0444 \\
\hline PPARG & rs 1801282 & $\mathrm{C}$ & $1.650(0.998-2.726)$ & 0.051 & 0.0056 \\
\hline
\end{tabular}

Notes: Odd ratios and P-values were adjusted for age, sex, BMI, SBP; DBP; TG; FBG; Smoke and Drink under the additive model. 
Table 3 Association of the PRS with AHI and OSA

\begin{tabular}{|c|c|c|c|c|c|c|}
\hline \multirow[t]{2}{*}{ PRS Category } & \multicolumn{2}{|l|}{ AHI } & \multicolumn{2}{|c|}{ OSA } & \multicolumn{2}{|c|}{ Moderate-to-Severe OSA } \\
\hline & $\beta(95 \% \mathrm{Cl})$ & $P$ value & OR $(95 \% \mathrm{CI})$ & $P$ value & OR (95\% Cl) & $P$ value \\
\hline Low risk & Reference & & Reference & & Reference & \\
\hline Intermediate risk & $5.349(-0.094-10.791)$ & 0.054 & $2.256(1.110-4.587)$ & 0.025 & $4.24 I(I .848-9.733)$ & 0.001 \\
\hline High risk & $7.00 \mathrm{I}(1.504-12.499) 0.013$ & & $2.84 I(I .374-5.874)$ & 0.005 & $5.372(2.299-12.55 \mid)$ & $<0.001$ \\
\hline Per SD & $1.241(0.025-2.457)$ & 0.046 & $1.237(1.021-1.499)$ & 0.030 & & \\
\hline
\end{tabular}

Notes: Risk estimates were adjusted for age, sex, BMI, SBP; DBP; TG; FBG; Smoke; Drink. PRS was constructed by summing the number of risk alleles among 9 SNPs shown in Table 2. PRS risk boundaries: low $<2$ ( $\leq 20$ th percentile); intermediate $\geq 2$ and $<7$ (20th-80th percentile); high $\geq 7$ ( $\geq 80$ th percentile).

Abbreviations: $\mathrm{OR}$, odd ratio; $\mathrm{Cl}$, confidence interval; $\mathrm{PRS}$, polygenic risk score.

To investigate whether increasing the number of risk alleles can increase the occurrence of OSA, we first developed a genetic risk score based on the nine SNPs. The total number of risk alleles in all subjects analyzed in the study ranged from 0 to 15. A higher PRS was significantly associated with an increased risk of OSA, independent of established clinical predictors, including age, sex, BMI, blood pressure, TG, FPG, smoking status, and drinking status (Table 3). An increase of 1 SD of the PRS was associated with a $24 \%$ increase in the odds of incident OSA (OR: 1.237; 95\% CI: $1.021-1.499, P=0.03$ ). Specifically, when evaluating participants in low, intermediate, and high genetic risk score categories, a gradient of risk for having OSA was evident. Those with higher PRS had a 2.84-fold (95\% CI: 1.374-5.874, $P=0.005)$ and 5.37-fold (95\% CI: $2.299-12.551, P<0.001)$ greater risk for OSA and moderate-to-severe OSA than those with lower genetic risk, respectively.

\section{Assessment of the PRS}

From our univariate and multivariate logistic regression analysis, we identified four independent risk factors for OSA: age $(\mathrm{OR}=1.043, P<0.001)$, male gender $(\mathrm{OR}=3.490, P<0.001)$, BMI $(\mathrm{OR}=1.293, P<0.001)$, and high risk in the $\mathrm{BQ}(\mathrm{OR}=2.085, P=0.004)$. These factors were all significantly associated with OSA (Table 4$)$.

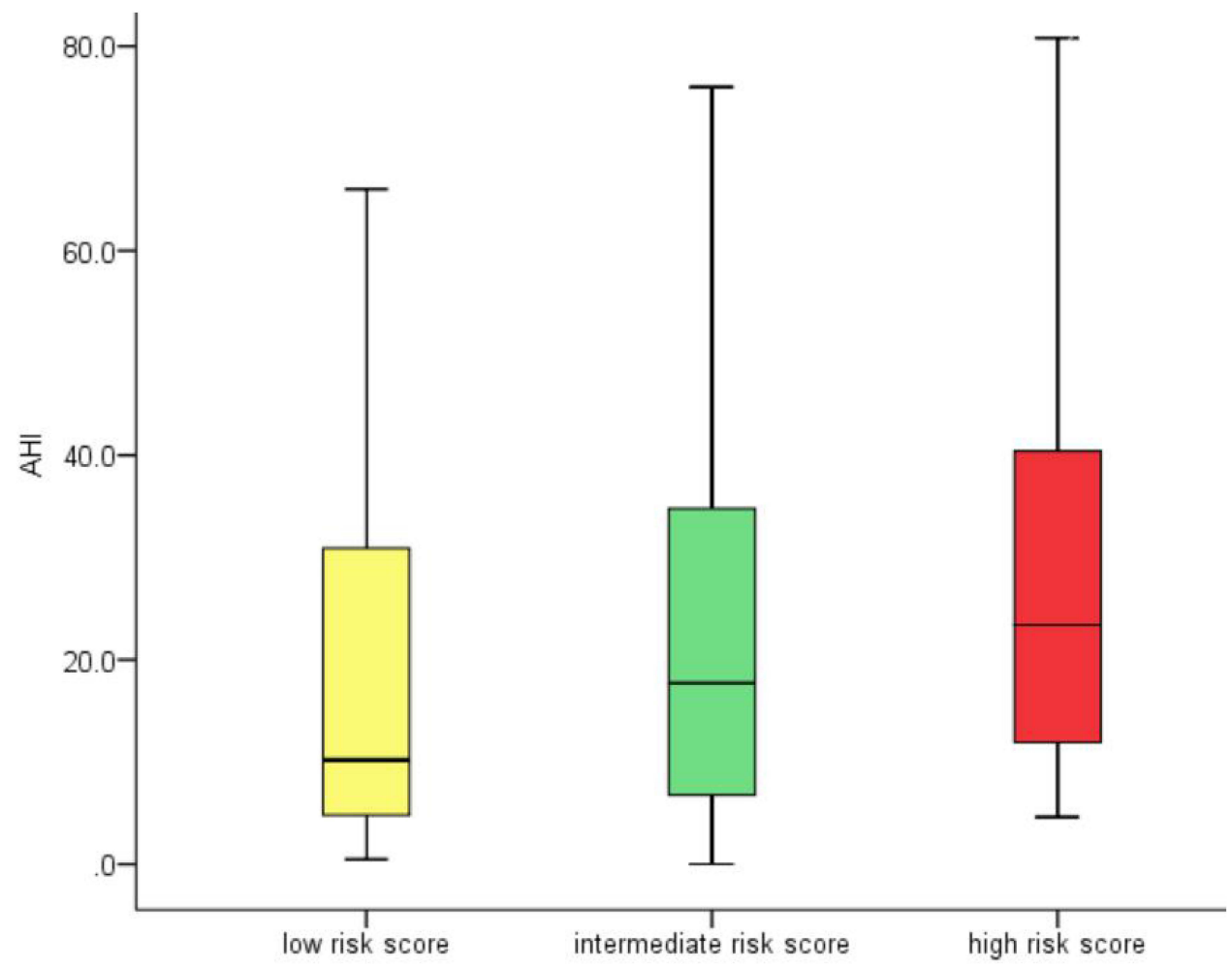

Figure I Association of PRS with AHI. 
Table 4 Univariable and Multivariable Logistic Regression Analysis for OSA

\begin{tabular}{|c|c|c|c|c|}
\hline \multirow[t]{2}{*}{ Characteristics } & \multicolumn{2}{|c|}{ Univariable Analysis } & \multicolumn{2}{|c|}{ Multivariable Analysi } \\
\hline & OR $(95 \% \mathrm{Cl})$ & $P$ value & OR $(95 \% \mathrm{Cl})$ & $P$ value \\
\hline Sex Female & Reference & & Reference & \\
\hline Male & $2.6(1.8-3.8)$ & $<0.001$ & $3.490(2.098-5.806)$ & $<0.001$ \\
\hline Age & $1.0(1.0-1.0)$ & 0.027 & $1.043(1.025-1.061)$ & $<0.001$ \\
\hline BMI & $1.3(1.2-1.3)$ & $<0.001$ & $1.293(1.219-1.371)$ & $<0.001$ \\
\hline SBP & $1.0(1.0-1.0)$ & 0.005 & $0.999(0.985-1.012)$ & 0.864 \\
\hline DBP & $1.0(1.0-1.0)$ & 0.004 & I.007 (0.987-I.027) & 0.492 \\
\hline HDL-C & $0.9(0.8-1.2)$ & 0.586 & $1.325(0.83 I-2.112)$ & 0.237 \\
\hline LDL-C & $1.0(0.8-1.2)$ & 0.928 & $0.885(0.64 I-I .222)$ & 0.458 \\
\hline TC & $1.0(0.9-1.2)$ & 0.530 & I.I27 (0.86I-I.476) & 0.383 \\
\hline TG & $1.3(1.1-1.6)$ & $<0.001$ & I. 170 (0.990-I.382) & 0.065 \\
\hline FBG & I.I (I.0-I.2) & 0.003 & $1.088(0.993-1.192)$ & 0.071 \\
\hline \multicolumn{5}{|l|}{ Drink } \\
\hline Yes & Reference & & Reference & \\
\hline No & $1.2(0.8-1.6)$ & 0.342 & $0.807(0.533-\mid .221)$ & 0.311 \\
\hline \multicolumn{5}{|l|}{ Smoke } \\
\hline Yes & Reference & & Reference & \\
\hline No & $1.4(1.0-1.9)$ & 0.066 & $1.040(0.685-1.579)$ & 0.854 \\
\hline ESS & I.I (I.0-I.I) & 0.007 & $0.996(0.952-1.043)$ & 0.866 \\
\hline \multicolumn{5}{|l|}{ Berlin Questionnaire } \\
\hline Low risk & Reference & & Reference & \\
\hline High risk & $3.4(2.3-5.2)$ & $<0.001$ & $2.085(1.266-3.436)$ & 0.004 \\
\hline
\end{tabular}

To predict disease status for OSA in a given individual, we constructed a prediction model on the basis of the PRS or the liability value calculated from PRS, as well as clinical risk factors. Figure 2 shows the ROC for OSA. In model 1, the conventional risk factors of age, sex, BMI, and BQ were included. The AUC for the conventional model was 0.75. Incorporating PRS into the model statistically significantly increased the AUC to $0.78(P=0.024)$. In moderate-to-severe OSA subjects, adding the PRS into model 1 increased the AUC from 0.80 to $0.83(P=0.017)$.

\section{Discussion}

OSA is a heritable polygenic disease. Obesity, craniofacial structure, neural control of upper airway muscles, and sleep and circadian rhythm are risk factors for OSA. Genes related to these factors contribute to the genetic spectrum of OSA. ${ }^{16}$ Obesity is an independent risk factor for OSA, which increases the risk of OSA by 10-14 times. Obesity, measured by BMI, is expected to explain up to $40 \%$ of genetic variation in AHI. ${ }^{17}$

$A D I P O Q, T N F$, and PPARG play very important roles in the process of lipid metabolism and obesity. They participate in the body's fat metabolism and body energy balance by affecting the expression of transcription factors in glucose metabolism, lipid metabolism, and adipocyte differentiation. They may increase the risk of OSA by increasing the risk of obesity. Previous studies found that all three genes, $A D I P O Q, T N F$, and PPARG, are associated with OSA. Importantly, the levels of circulating adiponectin were significantly different in patients with OSA compared with those in control subjects. ${ }^{18-20}$ Common variations in the $A D I P O Q$ gene were linked to an increased risk and severity of OSA. ${ }^{21}$ The $A D I P O Q$ gene encodes the hormone adiponectin, and its levels correlate negatively with BMI. ${ }^{22,23} T N F-\alpha$ gene expression levels were significantly higher in the OSA group than in the control group. ${ }^{24,25}$ Some studies suggested a relationship between polymorphic variation in the $T N F-\alpha$ gene and OSA. The $-308 \mathrm{G} / \mathrm{A}$ polymorphism has been associated with increased levels of $T N F-\alpha$, which is involved in intermittent hypoxia. ${ }^{25,26}$ Meta-analysis of three studies estimated that the $T N F-\alpha(-308 \mathrm{G} / \mathrm{A})$ polymorphism is a risk factor for OSA. ${ }^{27} \mathrm{~A}$ limitation of our study is that we did not include this SNP in our analysis. A previous study reported that the expression of PPARG was downregulated in the adipose tissue of OSA patients compared with controls. ${ }^{28}$ 
A

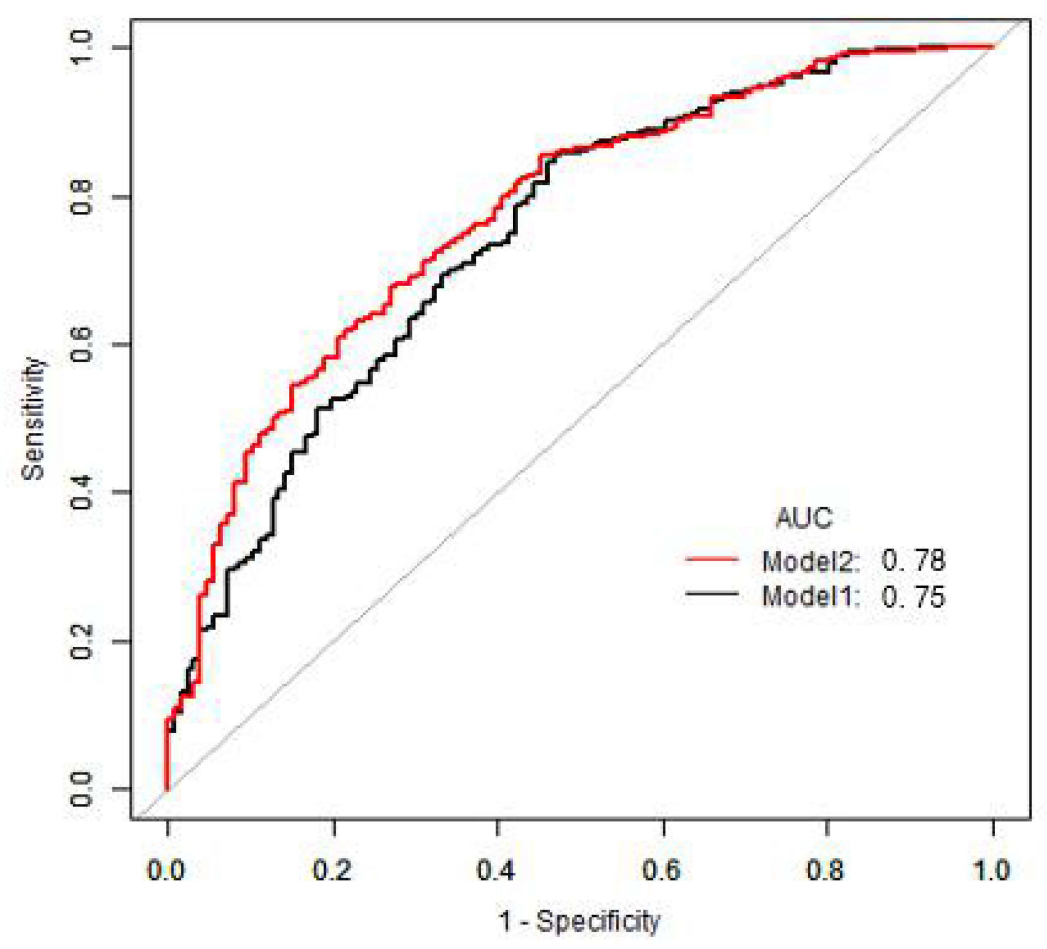

B

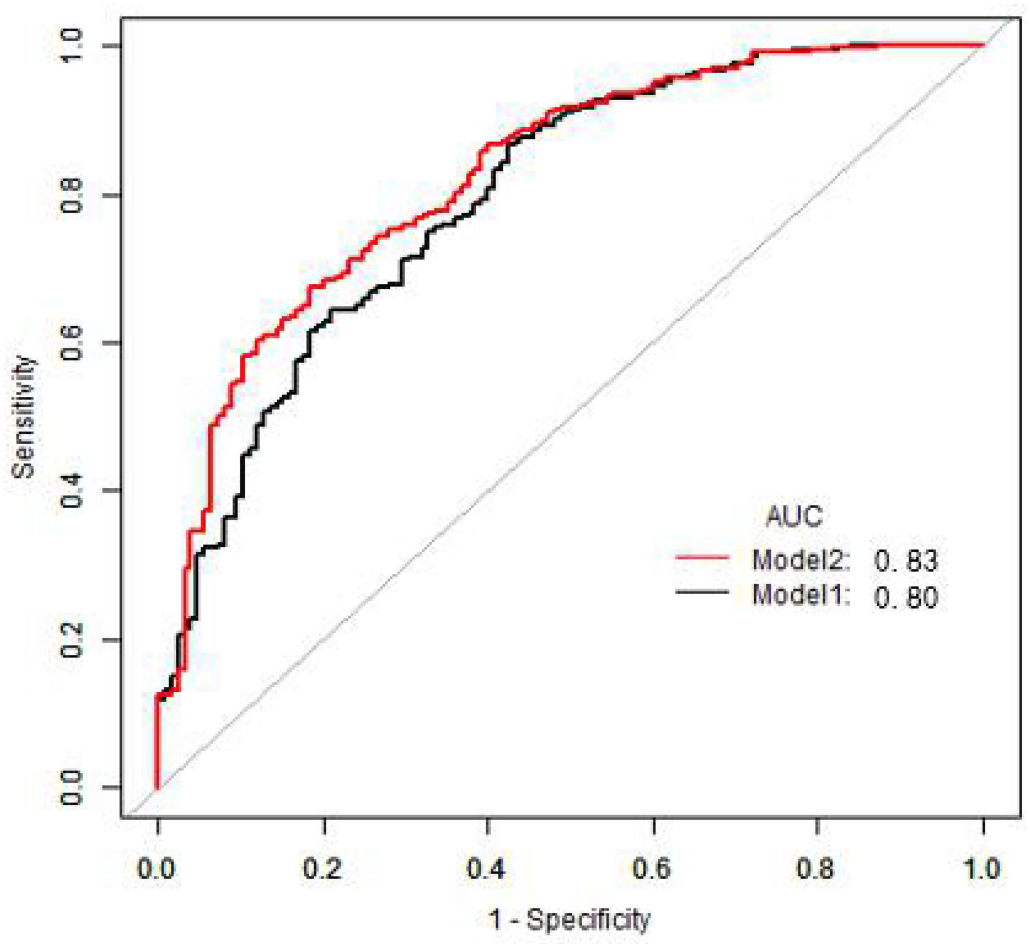

Figure 2 ROC curves for the prediction model on the basis of the number of risk alleles for OSA. (A) All OSA and (B) restricted to moderate-to-severe OSA. The prediction model for OSA was constructed using the logistic regression model, and ROC curves for the model were generated. In model I, only clinical risk factors (age, sex, $\mathrm{BMI}$ and Berlin questionnaire) were included, whereas the number of risk alleles was used as an independent variable together with age, sex, BMI and Berlin questionnaire as covariates in model 2. 
Furthermore, $P P A R G$ was positively correlated with the BMI and PPARG (rs1801282) with the allelic variants of Ala12Ala or Pro12Pro will most likely become overweight/obese. ${ }^{29,30} P P A R G$ can lead to obesity by increasing fatty acid absorption and fat accumulation. Consistent with those results, our previous research also found that $A D I P O Q, T N F$, and PPARG were associated with OSA risk. $A D I P O Q$ variants rs3774262, rs4686803, rs1063537, and rs2082940 and PPARG rs 1801282 were associated with the prevalence of OSA in Han Chinese individuals. ${ }^{10,11}$ The $T N F$ gene variants rs3093661, rs3093662, and rs3093664 were also associated with risk of OSA in the same dataset. Because of the importance of obesity in OSA, we therefore included these recently identified common variants of the PPARG, TNF, and ADIPOQ genes in this study, establishing a PRS and exploring the genetic architecture of OSA.

Recent studies have demonstrated the utility of PRS for disease risk stratification, as well as their implications for early disease detection, prevention, therapeutic intervention, and/or life planning. ${ }^{31}$ This is a robust approach in that it provides valuable information on the additive effects of multiple gene variants in the same individual. It has been used for risk scoring of many polygenic diseases, including cardiovascular disease, diabetes, and breast cancer, as well as neurological disorders.

In our study, we used the PRS approach to determine OSA susceptibility. We selected nine variants from adipokinepathway genes (TNF, PPARG, and $A D I P O Q)$, meaning that an individual could have a maximum of 18 risk alleles. By using a gene scoring approach, we found that SNPs in adipokine pathway genes may influence the development of OSA. Subjects with a higher PRS had a 2.84-fold greater risk of OSA than those with a lower genetic risk. Furthermore, this inclusive risk score provides significant improvement in the discrimination and prediction of OSA. The AUC increased significantly from 0.75 to 0.78 for OSA when genetic variants were combined with clinical risk factors. The AUC increased to 0.83 in Model 2, meaning that the PRS had a greater predictive effect on moderate-to-severe OSA. While the AUC of the PRS alone may appear modest, the predicted risk differences in the tails of the distribution are large.

Our results should be interpreted in the context of several potential limitations. Firstly, we performed the present association study in a cross-sectional setting; therefore, it is difficult to estimate the accurate predictive power of our genetic model. Secondly, there are many environmental factors affecting OSA, and this study only explores four common factors: gender, age, BMI, and BQ. Thirdly, we took a focused candidate gene approach to evaluate key SNPs, excluding additional genes that may contribute to OSA. Several reported OSA risk-associated SNPs from genome-wide association studies were not included in our study. Our study also has several strengths: i) it was large enough for key outcomes of interest, ii) we used statistical strategies to assess the robustness of associations, such as bootstrap resampling and discrimination improvement measures; and iii) subjects in this study were consecutively recruited to reduce the effects of outcome-selection bias.

\section{Conclusion}

In conclusion, the PRS we constructed here was significantly associated with an increased risk of OSA in a Chinese Han population. Furthermore, combining this PRS with conventional risk factors for OSA could improve the predictive performance. Although currently available genetic information remains insufficient for an accurate prediction of OSA, the information may be useful in groups with a particular clinical background, such as the obese population. Future work is necessary to identify the underlying functional or causal gene variants with potentially stronger effects, and updated analysis using these genes may further improve risk determination for OSA.

\section{Data Sharing Statement}

All the data from this manuscript are publicly available.

\section{Ethics Approval and Consent to Participate}

All procedures performed in studies involving human participants were in accordance with the ethical standards of the institutional and/or national research committee and with the 1964 Helsinki declaration and its later amendments or comparable ethical standards. All participants provided informed consent.

\section{Consent for Publication}

All the authors have accepted responsibility for the entire content of this submitted manuscript and approved submission. 


\section{Acknowledgments}

We thank all study participants and investigators for making this project possible. We thank Xiaolu Jiao, Yunyun Yang, and Huahui Yu for their assistance in data acquisition and analysis. We also thank Shaoping Nie, Ming Zhang, Wenli Cheng, and Jingyao Fan for their assistance in blood sample collection. Dr. Wei Yongxiang and Dr. Qin Yanwen contributed to designing the research plan and revising the manuscript. Their research interests include obstructive sleep apnea epidemiology, the genetics of obstructive sleep apnea, and obstructive sleep apnea-associated cardiovascular diseases. We thank J. Iacona, Ph.D., from Liwen Bianji (Edanz) for editing the English text of a draft of this manuscript.

\section{Author Contributions}

All authors made a significant contribution to the work reported, whether that is in the conception, study design, execution, acquisition of data, analysis and interpretation, or in all these areas; took part in drafting, revising or critically reviewing the article; gave final approval of the version to be published; have agreed on the journal to which the article has been submitted; and agree to be accountable for all aspects of the work.

\section{Funding}

This study was supported by the National Natural Science Foundation of China (Grant Nos. 81670331, 81970224, and 81870335) and the Beijing Natural Science Foundation (Grant No. 7192030) Beijing Key Laboratory of Upper Airway Dysfunction and Related Cardiovascular Diseases (No: BZ0377).

\section{Disclosure}

The authors declare that they have no conflicts of interest.

\section{References}

1. Loube DI, Gay PC, Strohl KP, Pack AI, White DP, Collop NA. Indications for positive airway pressure treatment of adult obstructive sleep apnea patients: a consensus statement. Chest. 1999;115(3):863-866. doi:10.1378/chest.115.3.863

2. Cade BE, Chen H, Stilp AM, et al. Genetic associations with obstructive sleep apnea traits in Hispanic/Latino Americans. Am J Respir Crit Care Med. 2016;194(7):886-897. doi:10.1164/rccm.201512-2431OC

3. Peppard PE, Young T, Barnet JH, Palta M, Hagen EW, Hla KM. Increased prevalence of sleep-disordered breathing in adults. Am J Epidemiol. 2013;177(9):1006-1014. doi:10.1093/aje/kws342

4. Peng J, Song J. Effects of CPAP on the transcriptional signatures in patients with obstructive sleep apnea via coexpression network analysis. $J$ Cell Biochem. 2019;120(6):9277-9290. doi:10.1002/jcb.28203

5. Kripke DF, Kline LE, Nievergelt CM, et al. Genetic variants associated with sleep disorders. Sleep Med. 2015;16(2):217-224. doi:10.1016/j. sleep.2014.11.003

6. Varvarigou V, Dahabreh IJ, Malhotra A, Kales SN. A review of genetic association studies of obstructive sleep apnea: field synopsis and meta-analysis. Sleep. 2011;34(11):1461-1468. doi:10.5665/sleep.1376

7. Zhao X, Xu H, Qian Y, et al. Abdominal obesity is more strongly correlated with obstructive sleep apnea than general obesity in China: results from two separated observational and longitudinal studies. Obes Surg. 2019;29(8):2535-2547. doi:10.1007/s11695-019-03870-z

8. Zhang DM, Pang XL, Huang R. Adiponectin, omentin, ghrelin, and visfatin levels in obese patients with severe obstructive sleep apnea. Biomed Res Int. 2018;2018:3410135. doi:10.1155/2018/3410135

9. Van Eyck A, Van Hoorenbeeck K, De Winter BY, Van Gaal L, De Backer W, Verhulst SL. Sleep-disordered breathing, systemic adipokine secretion, and metabolic dysregulation in overweight and obese children and adolescents. Sleep Med. 2017;30:52-56. doi:10.1016/j. sleep.2015.11.014

10. Yang Y, Yang S, Jiao X, et al. Targeted sequencing analysis of the adiponectin gene identifies variants associated with obstructive sleep apnoea in Chinese Han population. Medicine. 2019;98(16):e15219. doi:10.1097/MD.0000000000015219

11. Jiao X, Yang S, Yang Y, et al. Targeted sequencing analysis of PPARG identifies a risk variant associated with obstructive sleep apnea in Chinese Han subjects. Sleep Breath. 2020;24:167-174.

12. Juan L, Yang S, Jiao X, et al. Targeted sequencing analysis of the leptin receptor gene identifies variants associated with obstructive sleep apnoea in Chinese Han Population. Lung. 2019;197(5):577-584. doi:10.1007/s00408-019-00254-z

13. Berry RB, Budhiraja R, Gottlieb DJ, et al. Rules for scoring respiratory events in sleep: update of the 2007 AASM manual for the scoring of sleep and associated events. Deliberations of the sleep apnea definitions task force of the American academy of sleep medicine. J Clin Sleep Med. 2012;8 (5):597-619. doi:10.5664/jcsm.2172

14. Netzer NC, Stoohs RA, Netzer CM, Clark K, Strohl KP. Using the Berlin Questionnaire to identify patients at risk for the sleep apnea syndrome. Ann Intern Med. 1999;131(7):485-491. doi:10.7326/0003-4819-131-7-199910050-00002

15. Zhou BF. Effect of body mass index on all-cause mortality and incidence of cardiovascular diseases-report for meta-analysis of prospective studies open optimal cut-off points of body mass index in Chinese adults. Biomed Environ Sci. 2002;15(3):245-252. 
16. Mukherjee S, Saxena R, Palmer LJ. The genetics of obstructive sleep apnoea. Respirology. 2018;23(1):18-27. doi:10.1111/resp.13212

17. Patel SR, Larkin EK, Redline S. Shared genetic basis for obstructive sleep apnea and adiposity measures. Int J Obesity. 2008;32(5):795-800. doi:10.1038/sj.ijo.0803803

18. Sertogullarindan B, Komuroglu A, Ucler R, Gunbatar H, Sunnetcioglu A, Cokluk E. Betatrophin association with serum triglyceride levels in obstructive sleep apnea patients. Ann Thorac Med. 2019;14(1):63-68. doi:10.4103/atm.ATM_52_18

19. Zeng F, Wang X, Hu W, Wang L. Association of adiponectin level and obstructive sleep apnea prevalence in obese subjects. Medicine. 2017;96(32): e7784. doi:10.1097/MD.0000000000007784

20. Gonzalez FJ, Xie C, Jiang C. The role of hypoxia-inducible factors in metabolic diseases. Nat Rev Endocrinol. 2019;15(1):21-32. doi:10.1038/ s41574-018-0096-Z

21. Wu W, Li Z, Tang T, et al. Single nucleotide polymorphisms in adiponectin gene are not directly associated with increased risk of obstructive sleep apnea syndrome in a Chinese Han Population. ORL J Otorhinolaryngol Relat Spec. 2016;78(6):308-319. doi:10.1159/000454734

22. Kanu JS, Gu Y, Zhi S. Single nucleotide polymorphism rs3774261 in the AdipoQ gene is associated with the risk of coronary heart disease (CHD) in Northeast Han Chinese population: a case-control study. Lipids Health Dis. 2016;15(1):6. doi:10.1186/s12944-015-0173-4

23. Du SX, Lu LL, Liu Y, Dong QJ, Xuan SY, Xin YN. Association of adiponectin gene polymorphisms with the risk of coronary artery disease in patients with nonalcoholic fatty liver disease in a Chinese Han Population. Hepat Mon. 2016;16(7):e37388. doi:10.5812/hepatmon.37388

24. Ugur KS, Acar M, Ozol D, et al. Gene expression profiles of tumor necrosis factor-alpha and endothelin-1 in obstructive sleep apnea. $O R L$ J Otorhinolaryngol Relat Spec. 2019;81(1):16-23. doi:10.1159/000490353

25. Bhushan B, Guleria R, Misra A, Luthra K, Vikram NK. TNF-alpha gene polymorphism and TNF-alpha levels in obese Asian Indians with obstructive sleep apnea. Respir Med. 2009;103(3):386-392. doi:10.1016/j.rmed.2008.10.001

26. Riha RL, Brander P, Vennelle M, et al. Tumour necrosis factor-alpha (-308) gene polymorphism in obstructive sleep apnoea-hypopnoea syndrome. Eur Respir J. 2005;26(4):673-678. doi:10.1183/09031936.05.00130804

27. Zhong A, Xiong X, Xu H, Shi M. An updated meta-analysis of the association between tumor necrosis factor-alpha -308G/A polymorphism and obstructive sleep apnea-hypopnea syndrome. PLoS One. 2014;9(9):e106270. doi:10.1371/journal.pone.0106270

28. Gharib SA, Hayes AL, Rosen MJ, Patel SR. A pathway-based analysis on the effects of obstructive sleep apnea in modulating visceral fat transcriptome. Sleep. 2013;36(1):23-30. doi:10.5665/sleep.2294

29. Castellano-Castillo D, Denechaud P-D, Fajas L. Human adipose tissue H3K4me3 histone mark in adipogenic, lipid metabolism and inflammatory genes is positively associated with BMI and HOMA-IR. PLoS One. 2019;14(4):e0215083. doi:10.1371/journal.pone.0215083

30. Rodríguez-Pardo C, Segura A, Zamorano-León JJ. Decision tree learning to predict overweight/obesity based on body mass index and gene polymporphisms. Gene. 2019;699:88-93. doi:10.1016/j.gene.2019.03.011

31. Torkamani A, Wineinger NE, Topol EJ. The personal and clinical utility of polygenic risk scores. Nat Rev Genet. 2018;19(9):581-590. doi:10.1038/ s41576-018-0018-x

Nature and Science of Sleep is an international, peer-reviewed, open access journal covering all aspects of sleep science and sleep medicine, including the neurophysiology and functions of sleep, the genetics of sleep, sleep and society, biological rhythms, dreaming, sleep disorders and therapy, and strategies to optimize healthy sleep. The manuscript management system is completely online and includes a very quick and fair peer-review system, which is all easy to use. Visit http://www.dovepress.com/testimonials.php to read real quotes from published authors.

Submit your manuscript here: https://www.dovepress.com/nature-and-science-of-sleep-journal 